\title{
A characterization for all interval doubling schemes of the lattice of permutations
}

\author{
Nathalie Caspard \\ CERMSEM, Maison des Sciences Économiques, Université Paris 1-Panthéon-Sorbonne, 106-112, Bd de l'Hôpital, \\ 75647 Paris Cedex 13, France.
}

received March 30, 1999, revised June 23, 1999, accepted June 23, 1999.

\begin{abstract}
The lattice $\mathcal{S}_{n}$ of all permutations on a $n$-element set has been shown to be bounded [5], which is a strong constructive property characterized by the fact that $\mathcal{S}_{n}$ admits what we call an interval doubling scheme. In this paper we characterize all interval doubling schemes of the lattice $\mathcal{S}_{n}$, a result that gives a nice precision on the bounded nature of the lattice of permutations. This theorem is a direct corollary of two strong properties (Proposition 3 and Theorem 3 ) that are also given with their proofs.
\end{abstract}

Keywords: Permutations, lattice, bounded lattice, interval doubling schemes, arrow relations, linear extension, tableaux.

\section{Introduction}

Permutations on a $n$-element set play a fundamental role in numerous fields, especially in computer science and pure or applied mathematics. The main reason for the importance of permutations in applied mathematics is that they appear like a fundamental ordinal model since the set $\mathcal{S}_{n}$ of all permutations on a $n$-element set is clearly in a bijective correspondence with the set of all linear orders on a set with the same cardinality. Guilbaud and Rosenstiehl have proved in 1963 that the set of all permutations on a finite set is a lattice [15]: indeed the transpositions which reverse neighbours in a permutation (and which give rise, as a generating system, to the symmetric group $\mathcal{S}_{n}$ ) define the cover relation of an order relation $\leq \mathcal{S}_{n}$, the so-called weak Bruhat order on $\mathcal{S}_{n}$, which is a lattice (Björner [4]). The lattice $\left(\mathcal{S}_{n}, \leq_{\mathcal{S}_{n}}\right)$ of permutations, also called Permutoedron, has been studied by several authors (see e.g. Barbut and Monjardet [1], Chameni-Nembua [6], Le Conte de Poly-Barbut [16, 17], Edelman and Greene [11], Markowsky [19]). Recently, Duquenne and Cherfouh [10] (and, independently and in a more general context, Le Conte de Poly-Barbut [18]) have proved that this lattice is semidistributive. This property has been reinforced in [5] where it is proved that the lattice of permutations is bounded (see Day [8] for the origins of this concept and for a number of characterizations), which means that the Permutoedron can be obtained from the twoelement lattice $\mathcal{Q}=(\{0,1\}, \leq)$ by a (finite) sequence of doublings of convex sets (the definition of this constructive operation can be found for instance in [2] or in [14]). Another characterization of bounded lattices is expressed in terms of $A$-table, a (particularly rich lattice encoding) concept defined from the arrow relations. A lattice is bounded if and only if its $A$-table admits what we call an interval doubling 
scheme [14]. In [2], the authors have shown how all interval doubling schemes of a bounded lattice $L$ are in a 1-1 correspondence with all different ways to construct $L$ from the two-element lattice by the doubling operation mentioned above. These interval doubling schemes are also in a 1-1 correspondence with all linear extensions of the poset of join-irreducible congruences and with all linear extensions of the poset $J_{L}$ with respect to the $D-$ rank defined in [12] (see this reference and also [9] for more definitions and details on this subject). The aim of this paper is to provide a simple characterization of all interval doubling schemes of the lattice of permutations (Corollary 2). This characterization is obtained as a corollary of two results: Proposition 3 which characterizes the order relation between all join-irreducible permutations (and dually between all meet-irreducible permutations) in terms of arrow relations, and Theorem 3 that generalizes our characterization result to a larger class of lattices.

In the following section, we give all necessary notions on lattices and on the arrow relations, and provide some useful preliminaries about permutations. Section 3 presents the results with their proofs.

\section{Preliminaries}

A partially ordered set (or poset) $(L, \leq)$ is a lattice if any pair $\{x, y\}$ of elements of $L$ has a least upper bound (also called join) denoted by $x \vee y$ and a greatest lower bound (also called meet) denoted by $x \wedge y$. The order relation $\leq$ on $L$ is the transitive closure of the cover relation $\prec$ of $L$, which is defined by $x \prec y$ if $x<y$ and there exists no $z \in L$ such that $x<z<y$. We then say that $x$ is covered by $y$ (or $y$ covers $x$ ). An element $j$ (resp. $m$ ) of $L$ is a join- (resp. meet-) irreducible of $L$ if it cannot be obtained as the join (resp. meet) of elements of $L$ distinct from $j$ (resp. from $m$ ). Equivalently, an element $j$ (resp. $m$ ) of $L$ is a non-zero (resp. non-unit) join- (resp. meet-) irreducible if it covers (resp. is covered by) a unique element in $L$, which is then denoted by $j^{-}$(resp. $m^{+}$). The set of non-zero join- (resp. non-unit meet-) irreducibles of a lattice $L$ is denoted by $J_{L}-$ or $J$ if no confusion arises - (resp. $M_{L}$ or $M$ ). We will also identify any lattice $(L, \leq)$ with its underlying set $L$ and all lattices will be represented by their diagram, i.e. by the transitive reduction of their cover relation, directed from bottom to top.

For other definitions about lattices not recalled here, see for instance the books by Barbut and Monjardet [1], Birkhoff [3] or Davey and Priestley [7].

Let $N=\{1,2, \ldots, n\}$ be a set with $n$ elements. A permutation on $N$ is a bijection $\alpha$ on $N$ that we denote $\alpha=\alpha_{1} \ldots \alpha_{i} \ldots \alpha_{n}$. For $i=1, \ldots, n-1, \alpha_{i}$ and $\alpha_{i+1}$ are said adjacent in the permutation $\alpha$. We denote by $\mathcal{S}_{n}$ the set of all permutations on $N$. It is clear that $\mathcal{S}_{n}$ is in a bijective correspondence with the set of all linear orders on a set with cardinality $n$. According to this bijection, we will freely mix these two notions, considering a permutation $\alpha$ as the linear order $\alpha=\left\{\left(\alpha_{i}, \alpha_{j}\right): 1 \leq i<j \leq n\right\}$ and applying all ordinal notations and concepts to permutations. In the following, an ordered pair $\left(\alpha_{i}, \alpha_{j}\right)$ will rather be written $\alpha_{i} \alpha_{j}$.

For a permutation $\alpha$, we note $A(\alpha)$ the set of all agreements of $\alpha$, i.e. the set of all ordered pairs $\left(\alpha_{i}, \alpha_{j}\right)$ of $\alpha$ satisfying $\alpha_{i}<\alpha_{j}$. The set $D(\alpha)$ of all disagreements of $\alpha$ is defined as the set of all ordered pairs $\left(\alpha_{i}, \alpha_{j}\right)$ of $\alpha$ satisfying $\alpha_{j}<\alpha_{i}$. An order relation between the permutations of $\mathcal{S}_{n}$ is defined by $\alpha \leq \beta$ if $A(\beta) \subseteq A(\alpha)$ (or equivalently if $D(\alpha) \subseteq D(\beta)$ ). For this order relation (which equals the weak Bruhat order on the set of permutations), the set $\mathcal{S}_{n}$ of permutations is a lattice with the natural order $0_{\mathcal{S}_{n}}=1 \ldots k(k+1) \ldots n$ as least element and the dual order $1_{\mathcal{S}_{n}}=n \ldots k(k-1) \ldots 1$ as greatest element. The cover relation $\prec$ on $\mathcal{S}_{n}$ is defined by $\alpha \prec \beta$ if there exists a unique ordered pair $\left(\alpha_{i}, \alpha_{i+1}\right)$ of elements of $N$ that are adjacent in $\alpha$ and $\beta$ and that satisfy $\beta=\left[\alpha \backslash\left(\alpha_{i}, \alpha_{i+1}\right)\right] \cup\left(\alpha_{i+1}, \alpha_{i}\right)$. If a permutation 
$\alpha$ admits an agreement made of two adjacent elements $\alpha_{i}$ and $\alpha_{i+1}$ (i.e. $\alpha=\alpha_{1} \ldots \alpha_{i} \alpha_{i+1} \ldots \alpha_{n}$ with $\alpha_{i}<\alpha_{i+1}$ ), we say that $\alpha$ has a increasing (in $i$ ) and dually, if $\alpha$ admits a disagreement made of two adjacent elements $\alpha_{i}$ and $\alpha_{i+1}$ (i.e. $\alpha=\alpha_{1} \ldots \alpha_{i} \alpha_{i+1} \ldots \alpha_{n}$ with $\alpha_{i+1}<\alpha_{i}$ ), we say that $\alpha$ has an decreasing (in $i$ ).

The irreducible permutations are characterized by means of the increasings and decreasings as follows: a permutation is join-irreducible (resp. meet-irreducible) if and only if it admits a unique decreasing (resp. increasing).

Remark 1 Any join-irreducible permutation $\gamma$ can be denoted by $\gamma=A|\bar{A}=B v| u \bar{B}(u, v \in N)$, where $A=B v$ and $\bar{A}=u \bar{B}$ are the left and right factors of $\gamma$ compatible with the order $0_{\mathcal{S}_{n}}$, and where vu is the unique decreasing of $\gamma$. Dually, any meet-irreducible permutation $\mu$ will be written $\mu=D|\bar{D}=C l| p \bar{C}$, with $D=C l$ and $\bar{D}=p \bar{C}$ the left and right factors of $\mu$ compatible with the order $1_{\mathcal{S}_{n}}$ and where lp is the unique increasing of $\mu$. It is clear that a join-irreducible (resp. meet-irreducible) permutation is completely determined by $A$ or by $\bar{A}$ (resp. by $D$ or by $\bar{D})$.

Remark 2 The unique lower cover of a join-irreducible permutation $\gamma=A|\bar{A}=B v| u \bar{B}$ is the permutation $\gamma^{-}=B u v \bar{B}$, obtained by changing the unique decreasing vu of $\gamma$ into the increasing uv. Dually, the unique upper cover of the meet-irreducible permutation $\mu=D|\bar{D}=C l| p \bar{C}$ is the permutation $\mu^{+}=C p l \bar{C}$, obtained by changing the unique increasing lp of $\mu$ into the decreasing $p l$.

The following fact is well known:

Remark $3|J|=|M|\left(=2^{n}-(n+1)\right)$.

The original definition of bounded lattices can be found for instance in [12]. There exists a simple characterization of these lattices due to Day [8] and expressed in [14] in terms of arrow relations or equivalently, in terms of $A$-table, a very useful tool for the description of numerous properties on lattices. We start with the definition of these notions, introduced by Wille and the "Darmstadt School" (see [13] and [20]).

Definition 1 Let $j$ be a join-irreducible and $m$ a meet-irreducible of a lattice L. The three arrow relations $\uparrow, \downarrow$ and $\uparrow$ are defined as follows:

(a) $j \uparrow m$ if $m$ is maximal in $\{t \in L: j \not \leq t\}$.

(b) $j \downarrow m$ if $j$ is minimal in $\{t \in L: t \not \leq m\}$.

(c) $j \uparrow m$ if $j \uparrow m$ and $j \downarrow m$.

Equivalently, $j \uparrow m$ if and only if $j \not \leq m$ and $j \leq m^{+}$and, dually, $j \downarrow m$ if and only if $j \not \leq m$ and $j^{-} \leq m$.

Moreover it is useful to define the following "strict" arrow relations:

Definition 2 Let $L, j$ and $m$ be as above.

(d) $j \uparrow . m$ if $j \uparrow m$ and $j \not m$.

(e) $j \downarrow$. $m$ if $j \downarrow m$ and $j \forall m$.

It is obvious that relations $\leq, \uparrow, \uparrow$. and $\downarrow$. do not intersect. This allows us to define a fundamental concept of lattice theory, the A-table ( $A$ for Arrow) of a lattice (Wille and the "Darmstadt School", 1983). 
Definition 3 Let $(L, \leq)=L$ be a lattice and $J$ and $M$ its sets of join-irreducibles and of meet-irreducibles respectively. The $A$-table of $L-$ denoted by $A_{L}-$ is equal to the tuple $A_{L}=(J, M, \leq, \uparrow, \uparrow ., \downarrow$.).

According to its definition, the $A$-table of a lattice $L$ can always be described by a two-dimensional table, whose rows are indexed by the join-irreducibles of $L$, and whose columns are indexed by its meetirreducibles. Each cell $(j, m)$ in the $A$-table contains a cross $\times$ if $j \leq m$, and an arrow $\uparrow$., $\downarrow$., or $\uparrow$ according to the adequate case. The cell $(j, m)$ contains the symbol $\circ$ if $(j, m)$ does not satisfy any of the previous conditions.

Obviously there exists many ways to present the $A$-table of a lattice $L$, according to the orders chosen on $J$ and $M$ for the indexing of rows and columns of the $A$-table. This naturally leads to the simple and fundamental notion of tableau, that we define below:

Definition 4 A tableau $T$ of lattice $L$ is a triple $T=\left(A_{L}, \mathcal{L}_{J}, \mathcal{L}_{M}\right)$ where $A_{L}$ is the $A$-table of $L$ and $\mathcal{L}_{J}$ and $\mathcal{L}_{M}$ two linear orders respectively defined on $J$ and $M$ and defining (from the top to the bottom and from the left to the right) the two orders of the "rows " and of the "columns" of tableau $T$.

So the $A$-table of $L$ can be described by $(|J| ! \times|M| !)$ equivalent such tableaux and a tableau is completely determined by the two orders $\mathcal{L}_{J}$ and $\mathcal{L}_{M}$. Figure 1 shows (the diagram of) the lattice of permutations $\mathcal{S}_{4}$ and its $A$-table, given by a tableau.

Remark 4 Each row and each column of this tableau contains at least one $\uparrow$.

In the introduction, we have recalled that the Permutoedron is semidistributive. Let us recall that a lattice $L$ is said semidistributive if for all elements $x, y, z \in L, x \wedge y=x \wedge z$ implies $x \wedge y=x \wedge(y \vee z)$ and $x \vee y=x \vee z$ implies $x \vee y=x \vee(y \wedge z))$. The following characterization of these lattices is well known:

Proposition 1 A finite lattice $L$ is semidistributive if and only if the two following conditions are satisfied:

1. For any $j \in J$, there exists a unique $m \in M$ such that $j \uparrow m$.

2. For any $m \in M$, there exists a unique $j \in J$ such that $j \uparrow m$.

This result means that $L$ is semidistributive if and only if the $A$-table of $L$ contains a unique $\uparrow$ on each line and on each column. According to Remark 4, this fact implies that $J$ and $M$ have the same cardinality and that these two sets are in a bijective correspondence induced by the relation $\uparrow$. Theorem 2 gives the expression of this bijection in the case of the semidistributive lattice of permutations.

The characterization of bounded lattices given in Theorem 1 clearly shows that a bounded lattice is semidistributive. We begin with one definition.

Definition 5 Let $L$ be a semidistributive lattice and $T=\left(A_{L}, \mathcal{L}_{J}, \mathcal{L}_{M}\right)$ a tableau of its $A$-table. We say that $T$ is an interval doubling scheme of $L$ if it satisfies the two following conditions:

1. The $|J|$ double arrows $\uparrow$ are on the principal diagonal of $T$.

2. All arrows $\uparrow$. are below this diagonal and all arrows $\downarrow$. are above.

For an illustration of that definition see Figure 2.

Theorem 1 (Geyer, 1994) A lattice $L$ is bounded if and only if its $A$-table admits at least one interval doubling scheme. 


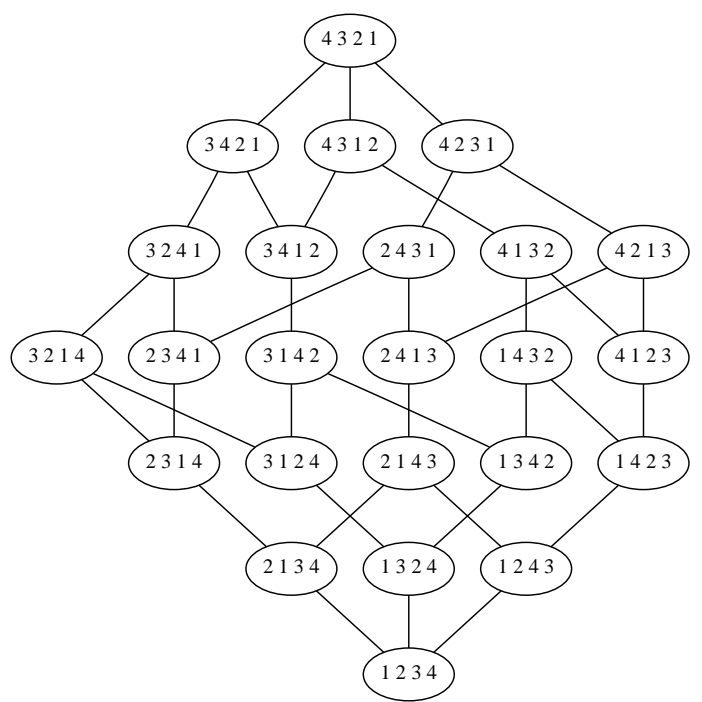

\begin{tabular}{|c|ccccccccccc|}
\hline$J \backslash M$ & 3421 & 4231 & 3241 & 2431 & 4312 & 4213 & 3214 & 2413 & 4132 & 3142 & 1432 \\
\hline 1243 & $\uparrow$ & $\times$ & $\downarrow$. & $\times$ & $\times$ & $\times$ & $\downarrow$ & $\times$ & $\times$ & $\downarrow$. & $\times$ \\
1324 & $\times$ & $\uparrow$ & $\times$ & $\downarrow$. & $\times$ & $\downarrow$ & $\times$ & $\downarrow$. & $\times$ & $\times$ & $\times$ \\
1342 & $\times$ & $\uparrow$. & $\uparrow$ & $\circ$ & $\times$ & $\circ$ & $\downarrow$ & $\circ$ & $\times$ & $\times$ & $\times$ \\
1423 & $\uparrow$. & $\times$ & $\circ$ & $\uparrow$ & $\times$ & $\times$ & $\circ$ & $\downarrow$. & $\times$ & $\circ$ & $\times$ \\
2134 & $\times$ & $\times$ & $\times$ & $\times$ & $\uparrow$ & $\times$ & $\times$ & $\times$ & $\downarrow$. & $\downarrow$. & $\downarrow$. \\
2314 & $\times$ & $\times$ & $\times$ & $\times$ & $\uparrow$. & $\uparrow$ & $\times$ & $\downarrow$ & $\circ$ & $\circ$ & $\circ$ \\
2341 & $\times$ & $\times$ & $\times$ & $\times$ & $\uparrow$. & $\uparrow$ & $\downarrow$ & $\circ$ & $\circ$ & $\circ$ & $\circ$ \\
2413 & $\uparrow$. & $\times$ & $\circ$ & $\times$ & $\uparrow$. & $\times$ & $\circ$ & $\uparrow$ & $\circ$ & $\circ$ & $\circ$ \\
3124 & $\times$ & $\uparrow$. & $\times$ & $\circ$ & $\times$ & $\circ$ & $\times$ & $\circ$ & $\uparrow$ & $\times$ & $\downarrow$. \\
3412 & $\times$ & $\uparrow$. & $\uparrow$. & $\circ$ & $\times$ & $\circ$ & $\circ$ & $\circ$ & $\uparrow$. & $\uparrow$ & $\circ$ \\
4123 & $\uparrow$. & $\times$ & $\circ$ & $\uparrow$. & $\times$ & $\times$ & $\circ$ & $\circ$ & $\times$ & $\circ$ & $\uparrow$ \\
\hline
\end{tabular}

Fig. 1: The lattice $\mathcal{S}_{4}$ of permutations and (a tableau of) its $A$-table.

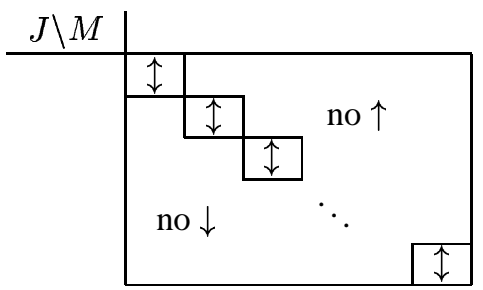

Fig. 2: An interval doubling scheme 
Clearly, the tableau in Figure 1 is an interval doubling scheme of the lattice $\mathcal{S}_{4}$. It is completely determined by the chosen linear order on $J$ (which is the lexicographical order on $J$ ) since, for this linear order, there exists a unique linear order on $M$ allowing to get all $\uparrow$ of the $A$-table on its principal diagonal. In [5], the author has shown the bounded nature of the Permutoedron by showing that the unique tableau given by the lexicographical order on $J$ and allowing the alignment of the $\downarrow$ on the diagonal is an interval doubling scheme of $\mathcal{S}_{n}$. Our aim here is to characterize all interval doubling schemes of the Permutoedron. To do so, we use the following characterization of the arrow relations in the lattice $\mathcal{S}_{n}$ (whose proof, easy, is left to the reader):

Proposition 2 Let $\gamma=B v \mid u \bar{B}$ be a join-irreducible and $\mu=C l \mid p \bar{C}$ a meet-irreducible of the lattice $\mathcal{S}_{n}$.

1. $\gamma \leq \mu \Longleftrightarrow D(\gamma) \subseteq D(\mu) \Longleftrightarrow A(\mu) \subseteq A(\gamma)$.

2. $\gamma \uparrow \mu \Longleftrightarrow p l \in D(\gamma)$ and $D(\gamma) \subseteq D\left(\mu^{+}\right)$.

3. $\gamma \downarrow \mu \Longleftrightarrow u v \in A(\mu)$ and $A(\mu) \subseteq A\left(\gamma^{-}\right)$.

4. $\quad \gamma \uparrow \mu \Longleftrightarrow p l \in D(\gamma), u v \in A(\mu), D(\gamma) \subseteq D\left(\mu^{+}\right)$and $A(\mu) \subseteq A\left(\gamma^{-}\right)$.

\section{The results}

We have seen that a semidistributive lattice is characterized by the existence of a unique $\uparrow$ on each line and on each column of its $A$-table, this relation $\uparrow$ inducing in the lattice a bijective correspondence between the sets $J$ and $M$. Without proof, we give the following theorem and corollary which make this bijection explicit in the case of the Permutoedron. For the proof see [5].

Theorem 2 1. Let $\gamma=B v \mid u \bar{B}$ be a join-irreducible and $\mu$ a meet-irreducible of lattice $\mathcal{S}_{n}$. Then

$$
\gamma \uparrow \mu \Longleftrightarrow \mu=C u \mid v \bar{C} \text { with }\left\{\begin{array}{l}
C=(\{x \in B: u<x\} \cup\{x \in \bar{B}: v<x\},>) \\
\bar{C}=(\{x \in B: x<u\} \cup\{x \in \bar{B}: x<v\},>)
\end{array}\right.
$$

2. Let $\mu=C l \mid p \bar{C}$ be a meet-irreducible and $\gamma$ a join-irreducible of lattice $\mathcal{S}_{n}$. Then

$$
\gamma \uparrow \mu \Longleftrightarrow \gamma=B p \mid l \bar{B} \quad \text { with }\left\{\begin{array}{l}
B=(\{x \in C: x<p\} \cup\{x \in \bar{C}: x<l\},<) \\
\bar{B}=(\{x \in C: p<x\} \cup\{x \in \bar{C}: l<x\},<)
\end{array}\right.
$$

Corollary 1 The relation $\uparrow$ induces a bijection between the sets of join-irreducibles and meet-irreducibles of $\mathcal{S}_{n}$ (semidistributivity of $\mathcal{S}_{n}$ ).

Definition 6 In a semidistributive lattice, the meet-irreducible image of the join-irreducible element $j$ in the bijection $\uparrow$ will be denoted by $m_{j}$ and conversely, the join-irreducible image of the meet-irreducible element $m$ will be denoted by $j_{m}$.

We shall now prove a strong property of the arrow relations in the lattice of permutations, that will be used to characterize all interval doubling schemes of $\mathcal{S}_{n}$.

Proposition 3 Let $\gamma$ be a join-irreducible of $\mathcal{S}_{n}$ and $\mu_{\gamma}$ its image in the bijection $\uparrow$. The following assertions are satisfied: 
1. $\forall \gamma^{\prime} \in J,\left(\gamma^{\prime} \uparrow . \mu_{\gamma} \Longleftrightarrow \gamma<\mathcal{S}_{n} \gamma^{\prime}\right)$.

2. $\forall \mu^{\prime} \in M,\left(\gamma_{\mu} \downarrow . \mu^{\prime} \Longleftrightarrow \mu^{\prime}<\mathcal{S}_{n} \mu\right)$.

Proof: To prove point 1, we set:

$$
\left\{\begin{array}{l}
\gamma=B v \mid u \bar{B} \\
\mu_{\gamma}=C u \mid v \bar{C} \\
\mu_{\gamma}^{+}=C v u \bar{C} \\
\gamma^{\prime}=B^{\prime} s \mid r \bar{B}^{\prime}
\end{array}\right.
$$

We then consider the following conditions:

$$
\left\{\begin{array}{l}
\text { (a) } v u \in D\left(\gamma^{\prime}\right) \\
\text { (b) } \forall x \in B,\left(u<x \Longrightarrow x \in B^{\prime}\right) \\
\text { (c) } \forall x \in \bar{B},\left(x<v \Longrightarrow x \in \bar{B}^{\prime}\right)
\end{array}\right.
$$

and we prove the equivalence of the three following conditions:

$$
\left\{\begin{array}{l}
(i) \gamma<\mathcal{S}_{n} \gamma^{\prime} \\
\text { (ii) conditions }(a),(b) \text { and }(c) \text { are satisfied } \\
\left(\text { iii) } \gamma^{\prime} \uparrow . \mu_{\gamma}\right.
\end{array}\right.
$$

$(i) \Longrightarrow(i i): \gamma<_{\mathcal{S}_{n}} \gamma^{\prime}$ implies $v u \in D\left(\gamma^{\prime}\right)$. Condition (a) is satisfied and implies $v \in B^{\prime} s$ and $u \in r \bar{B}^{\prime}$. Let $x \in B$ be such that $u<x$. So we have $x u \in D(\gamma)$, hence $x u \in D\left(\gamma^{\prime}\right)$. Then if $x \in r \bar{B}^{\prime}$, we have $u x \in A\left(\gamma^{\prime}\right)$, which is impossible. If $x=s$, then $v \in B^{\prime} s$ implies $v \leq s$, and so $x=s \in v u \bar{B}$, which contradicts $x \in B$. Finally, $x \in B^{\prime}$ and condition (b) is satisfied. Now for condition (c), let us consider $x \in \bar{B}$ such that $x<v$. We have $v x \in D(\gamma)$ and so $v x \in D\left(\gamma^{\prime}\right)$. If $x \in B^{\prime} s$ then $x v \in A\left(\gamma^{\prime}\right)$, which is impossible. If $x=r$ then $u \in r \bar{B}^{\prime}$ implies $r \leq u$, hence $x=r \in B v u$, which contradicts $x \in \bar{B}$. Finally, $x \in \bar{B}^{\prime}$ and condition (c) is satisfied.

$(i i) \Longrightarrow($ iii $)$ : Let us suppose that all conditions (a), (b) and (c) are satisfied. We prove that this implies $\left(B^{\prime} s \mid r \bar{B}^{\prime}=\right) \gamma^{\prime} \uparrow \mu_{\gamma}(=C u \mid v \bar{C})$, i.e. (Proposition 2) that we have $v u \in D\left(\gamma^{\prime}\right)$ and $A\left(\mu_{\gamma}^{+}\right) \subseteq$ $A\left(\gamma^{\prime}\right)$. Condition (a) gives $v u \in D\left(\gamma^{\prime}\right)$. It remains to prove that $A\left(\mu_{\gamma}^{+}\right) \subseteq A\left(\gamma^{\prime}\right)$. Let us compute the set $A\left(\mu_{\gamma}^{+}\right)$of all agreements of $\mu_{\gamma}^{+}=C v u \bar{C}$ :

$A\left(\mu_{\gamma}^{+}\right)=\{x v: x \in C$ and $x<v\} \cup\{u x: x \in \bar{C}$ and $u<x\} \cup\{x y: x \in C, y \in \bar{C}$ and $x<y\}$. We now prove that $A\left(\mu_{\gamma}^{+}\right) \subseteq A\left(\gamma^{\prime}\right)$.

- $\{x v: x \in C$ and $x<v\}$ : we have $u<x$ since $x \in C$ and on the other hand, $x \in C$ and $x<v$ imply (Theorem 2) $x \in B$. Thus condition (b) implies $x \in B^{\prime}$. Since $v \in B^{\prime} s$ (by condition (a)) and $x<v$, we obtain $x v \in A\left(\gamma^{\prime}\right)$.

- $\{u x: x \in \bar{C}$ and $u<x\}$ : we have $x<v$ since $x \in \bar{C}$ and on the other hand, $x \in \bar{C}$ and $u<x$ imply $x \in \bar{B}$ (Theorem 2). Thus condition (c) gives $x \in \bar{B}^{\prime}$ and, since $u \in r \bar{B}^{\prime}$ by condition (a), we have $u x \in A\left(\gamma^{\prime}\right)$. 
- $\{x y: x \in C, y \in \bar{C}$ and $x<y\}: x \in C, y \in \bar{C}$ and $x<y$ imply $u<x<y<v$. So we have on one hand $x \in C$ and $x<v$ that lead to $x \in B$ (Theorem 2) and since $u<x$, we obtain $x \in B^{\prime}$ according to (b). On the other hand, we have $y \in \bar{C}$ and $u<y$ that lead to $y \in \bar{B}$ and since $y<v$, we obtain $y \in \bar{B}^{\prime}$ by condition (c). Finally, $x \in B^{\prime}, y \in \bar{B}^{\prime}$ and $x<y$ and so $x y \in A\left(\gamma^{\prime}\right)$.

$($ iii $) \Longrightarrow($ i) : This fact trivially holds in any semidistributive lattice.

We have just given the proof of point 1. of the proposition. The proof of point 2. is dual.

Before we establish the characterization result for all interval doubling schemes of the Permutoedron (Corollary 2) describing all possible constructions of $\mathcal{S}_{n}$ by doublings of convex sets starting from the two-element lattice (details on this subject are given in [2]), we first prove the more general theorem below.

For a semidistributive lattice $L$ and a linear order $\mathcal{L}_{J}$ on $J$, we denote by $\mathcal{L}_{M}^{*}$ the unique linear order on $M$ such that the tableau $T=\left(A_{L}, \mathcal{L}_{J}, \mathcal{L}_{M}^{*}\right)$ has all $\uparrow$ on the principal diagonal.

Theorem 3 Let $L$ be a semidistributive lattice satisfying conditions 1. and 2. of Proposition 3 and let $\mathcal{L}_{J}$ be a linear order on the join-irreducible elements of $L$. The following conditions are equivalent:

1. $T=\left(A_{L}, \mathcal{L}_{J}, \mathcal{L}_{M}^{*}\right)$ is an interval doubling scheme of lattice $L$.

2. $\mathcal{L}_{J}$ is a linear extension of $\left(J, \leq_{L}\right)$ and $\mathcal{L}_{M}^{*}$ is a linear extension of $\left(M, \geq_{L}\right)$.

Proof: $1 . \Longrightarrow 2$ : Let $\mathcal{L}_{J}$ be a linear order on $J$. The tableau $T=\left(A_{L}, \mathcal{L}_{J}, \mathcal{L}_{M}^{*}\right)$ satisfies the alignment of the $\uparrow$ on the principal diagonal. If $\mathcal{L}_{J}$ is not a linear extension of $\left(J, \leq_{L}\right)$, there exists $j, j^{\prime} \in J$ such that $j \mathcal{L}_{J} j^{\prime}$ and $j^{\prime}<_{L} j$. But $j^{\prime}<_{L} j$ implies $j \uparrow . m_{j^{\prime}}$ (Proposition 3). Thus, $j$ and $j^{\prime}$ satisfy $j \mathcal{L}_{J} j^{\prime}$ and $j \uparrow . m_{j^{\prime}}$, and $T$ is not an interval doubling scheme.

Dually, we would show that a tableau $T=\left(A_{L}, \mathcal{L}_{J}, \mathcal{L}_{M}^{*}\right)$ is not an interval doubling scheme as soon as $\mathcal{L}_{M}^{*}$ is not a linear extension of $\left(M, \geq_{L}\right)$.

$2 . \Longrightarrow 1$ : Let $\mathcal{L}_{J}$ be a linear extension of $\left(J, \leq_{L}\right)$. Let us assume that the tableau $T=\left(A_{L}, \mathcal{L}_{J}, \mathcal{L}_{M}^{*}\right)$ is not an interval doubling scheme. So there exists $j, j^{\prime} \in J$ such that $j \mathcal{L}_{J} j^{\prime}$ and $\left(j \uparrow . m_{j^{\prime}}\right.$ or $j^{\prime} \downarrow$. $\left.m_{j}\right)$. In this case, $\mathcal{L}_{M}^{*}$ is not a linear extension of $\left(M, \geq_{L}\right)$. Indeed, $j \mathcal{L}_{J} j^{\prime}$ implies $j^{\prime} \nless_{L} j$ by hypothesis. But this last condition implies $j$ ․ $m_{j^{\prime}}$ (Proposition 3). So we have $j^{\prime} \downarrow$. $m_{j}$, which, according to the same proposition, implies $m_{j}<_{L} m_{j^{\prime}}$ and, since $j \mathcal{L}_{J} j^{\prime}$ implies $m_{j} \mathcal{L}_{M}^{*} m_{j^{\prime}}, \mathcal{L}_{M}^{*}$ is not a linear extension of $\left(M, \geq_{L}\right)$.

The announced result is now a direct corollary of Proposition 3 and Theorem 3.

Corollary 2 (Characterization) Let $\mathcal{L}_{J}$ be a linear order on the join-irreducible permutations of the Permutoedron. The following conditions are equivalent:

1. $T=\left(A_{\mathcal{S}_{n}}, \mathcal{L}_{J}, \mathcal{L}_{M}^{*}\right)$ is an interval doubling scheme of lattice $\mathcal{S}_{n}$.

2. $\mathcal{L}_{J}$ is a linear extension of $\left(J, \leq \mathcal{S}_{n}\right)$ and $\mathcal{L}_{M}^{*}$ is a linear extension of $\left(M, \geq \mathcal{S}_{n}\right)$. 

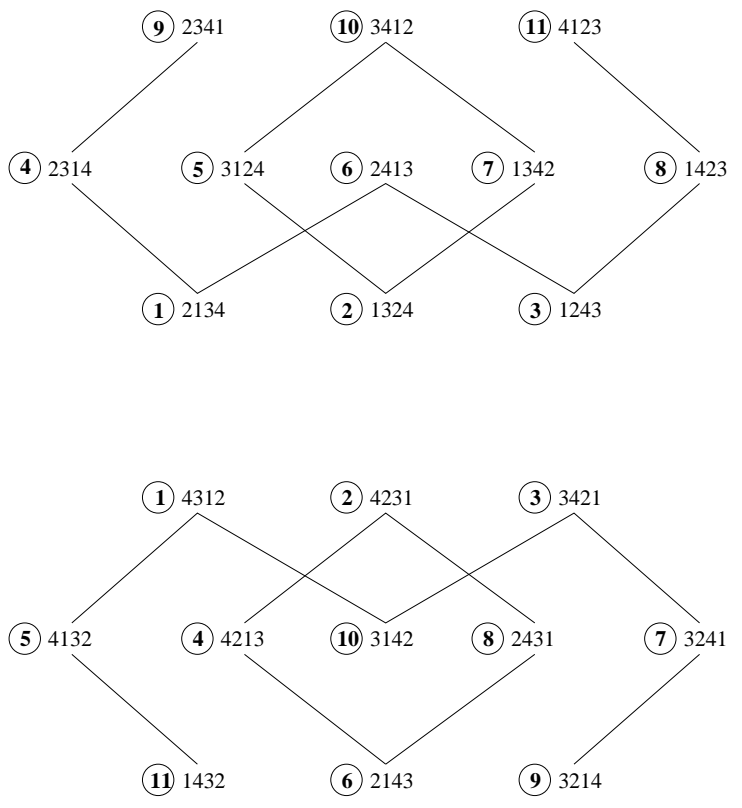

Fig. 3: A linear extension $\mathcal{L}_{J}$ of $\left(J, \leq \mathcal{S}_{4}\right)$ for which the linear order $\mathcal{L}_{M}^{*}$ on $M$ is not a linear extension of $\left(M, \geq \mathcal{S}_{4}\right)$.

Figure 3 shows that for a given linear extension $\mathcal{L}_{J}$ of the join-irreducibles of lattice $\mathcal{S}_{4}$, the linear order $\mathcal{L}_{M}^{*}$ on the meet-irreducibles is not necessarily a linear extension of $\left(M, \geq_{\mathcal{S}_{n}}\right)$ and so the theorem is not trivial. Indeed, if we numerate the join-irreducible permutations from 1 to 11 as shown on the figure, a linear extension of $\left(J, \leq_{\mathcal{S}_{4}}\right)$ is then: $1<2<3<4<5<6<7<8<9<10<11$, whereas the corresponding linear order $\mathcal{L}_{M}^{*}$ on $M$ is not a linear extension of $\left(M, \geq \mathcal{S}_{n}\right)$ (observe permutations 6 and 8).

\section{Conclusion}

We have recalled that all interval doubling schemes of a bounded lattice $L$ are in bijection with all different ways to construct $L$ starting from the two-element lattice by doublings of convex sets. The result of characterization of all interval doubling schemes of the Permutoedron appears therefore like a significant step towards a better understanding of the constructive properties of this lattice. Moreover, in [5], the author has conjectured that all (finite) Coxeter lattices (containing the infinite family of lattices $\left(\mathcal{S}_{n}\right)_{n \geq 2}$ ) could themselves be bounded lattices. In this case, the question would arise to know if there exists a similar characterization result about the interval doubling schemes of these lattices.

At last, we have proved Proposition 3 in a particular class of lattices (semidistributive lattices satisfying both conditions of Proposition 3) and we are interested to determine if this class is maximal for this property on the arrow relations. 


\section{Acknowledgements}

I want to thank Professor Bernard Monjardet for fruitful discussions and helpul remarks. I also thank referees for their helpful remarks.

\section{References}

[1] M. Barbut and B. Monjardet, Ordre et classification, vols. 1 and 2, Hachette, Paris, France (1970).

[2] K. Bertet and N. Caspard, Doubling convex sets in lattices: characterizations and recognition algorithms, preprint. Submitted.

[3] G. Birkhoff, Lattice Theory (first ed.), Amer. Math.Soc., Providence (1940).

[4] A. Björner, Orderings of Coxeter groups, Contemp. Math., Amer. Math. Soc. Providence, R. I., (1984), vol. 34, 175-195.

[5] N. Caspard, The lattice of permutations is bounded, preprint. Submitted.

[6] C. Chameni-Nembua, Permutoèdre et choix social, Thèse de Mathématiques Appliquées aux Sciences Sociales, Université Paris 5 (1989).

[7] B.A. Davey and H.A. Priestley, Introduction to Lattices and Order, Cambridge University Press (1992).

[8] A. Day, Characterizations of finite lattices that are bounded-homomorphic images or sublattices of free lattices, Canadian J. Math. 31 (1979), 69-78.

[9] A. Day, Congruence normality: the characterization of the doubling class of convex sets, Algebra Universalis 31 (1994), 397-406.

[10] V. Duquenne and A. Cherfouh, On permutation lattices, Math. Soc. Sciences 27 (1994), 73-89.

[11] P. Edelman and C. Greene, Combinatorial correspondences for Young tableaux , balanced tableaux and maximal chains in the weak Bruhat order of $\mathcal{S}_{n}$, Combinatorics and Algebra, C. Greene (ed.), Contemporary Mathematics 34 American Mathematical Society, Providence, R.I. (1984), 155-162.

[12] R. Freese, J. Jezek and J.B. Nation, Free lattices, Mathematical Surveys and Monographs, vol. 42, American Mathematical Society, (1991).

[13] B. Ganter and R. Wille, Formale Begriffsanalyse: Mathematische Grundlagen (1997) Springer Verlag, Berlin (english translation 1999).

[14] W. Geyer, The generalized doubling construction and formal concept analysis, Algebra Universalis 32 (1994), 341-367.

[15] G. Guilbaud and P. Rosenstiehl, Analyse algébrique d'un scrutin, Math. Sci. Hum. 4 (1963), 9-33.

[16] C. Le Conte de Poly-Barbut, Automorphismes du permutoèdre et votes de Condorcet, Math. Inf. Sci. hum. 111 (1990), 73-82. 
[17] C. Le Conte de Poly-Barbut, Le diagramme du treillis permutoèdre est intersection des diagrammes de 2 produits directs d'ordres totaux, Math. Inf. Sci. Hum. 112 (1990), 49-53.

[18] C. Le Conte de Poly-Barbut, Sur les treillis de Coxeter finis, Math. Inf. Sci. Hum., 125 (1994), 41-57.

[19] G. Markowsky, Permutation Lattices Revisited, Math. Soc. Sciences 27 (1994), 59-72.

[20] R. Wille, Subdirect decomposition of concept lattices, Algebra Universalis 17 (1983), 275-287. 
